\title{
A MULTI STAGE HEURISTIC FOR MANUFACTURING CELL FORMATION
}

\author{
P.Krishnananda Rao \\ Professor, Department of Mechanical and Manufacturing Engineering, Manipal Institute of Technology, \\ Karnataka, India
}

\begin{abstract}
Cellular manufacturing (CM) is a modern manufacturing concept based on the Group technology (GT) philosophy. CM enables a manufacturing unit to enhance its productivity substantially through improved utilization of resources. In Cellular manufacturing system design process the task of solving cell formation problem (machine-component grouping) is the central issue. Cell formation problem is considered as complex one due to its combinatorial nature. Research interest is growing towards development of novel solution methodologies for the purpose of cell formation. Even though numerous approaches have been implemented by researchers for the task of machine-component grouping, the effectiveness of multi stage solution procedures based on the integration of traditional and non-traditional optimization techniques needs to be investigated adequately. This paper demonstrates the development and implementation of a multi stage heuristic based on the integration of traditional cluster analysis technique and non- traditional simulated annealing technique for standard cell formation. The proposed heuristic is tested using a number of binary and non-binary datasets and computational results are presented. Results indicate that proposed heuristic is capable of generating promising quality solutions when compared with a few alternate cell forming approaches.
\end{abstract}

Key Words: Cellular manufacturing, Group technology Cell formation, Cellular manufacturing system, Cluster analysis, Simulated annealing.

\section{INTRODUCTION}

In the present competitive business environment manufacturing industries are striving to implement novel strategies to enhance their profitability and productivity for survival and growth. Cellular manufacturing (CM) is a novel manufacturing concept which has proved to be effective in improving the productivity of an enterprise. $\mathrm{CM}$ is an application of Group technology (GT) philosophy. GT exploits similarities between objects and groups similar objects into homogeneous clusters. In the context of manufacturing, GT helps in the identification of dissimilar machine groups and part (component) families for processing on each machine group so as to practice CM. The task of grouping machines into cells and assigning part families to each cell is popularly known as cell formation. Cell formation is regarded as the first and the complex step in Cellular manufacturing system (CMS) design process. Due to its combinatorial nature, research interest is growing towards implementation of novel and efficient solution methodologies for manufacturing cell formation. Two types of cell formation problem which have been investigated by researchers are binary and generalized cell formations. In binary cell formation machine groups and respective part families are formed using the processing information of parts present in binary (0/1) machine-component incidence matrix (MCIM). In binary MCIM, entrry ' 1 ' indicates the requirement of a specific machine by a specific part and ' 0 ' indicates the opposite. In generalized cell formation the machine groups and the corresponding part families are formed using the non-binary non binary MCIM containing the processing information parts and their production data such as processing times, production volumes, operation sequences, alternate process routings, production costs, etc. Binary cell formation offers rough cut solutions whereas generalized cell formation offers realistic machine component groupings considering the real time production environment.

Numerous approaches have been introduced the researchers over the years towards solving the manufacturing cell formation problem. A review of cell forming approaches is carried out by Grammatoula and Wilson [1], Arora et al. [2]. Production flow analysis technique was applied by Burbidge [3] for machine and component grouping utilizing MCIM with binary data. Chandrasekharan and Rajagopalan [4] introduced array based methods for cell formation. Similarity coefficient based cell forming approaches have been put forth by McAuley [5] and Gupta and Seifoddini [6]. Chandrasekharan and Rajagopalan [7] and Srinivasan and Narendran [8] introduced non- hierarchical clustering approaches namely ZODIAC and GRAFICS for machinecomponent grouping. These approaches require fixation of total number of cells to be formed in advance. Srinivasan et al. [9], Paydar and Sahebjamnia [10] and Mahdavi et al.[11] demonstrated mathematical programming based approaches for machine-component cell formation. Even though mathematical programming based approaches can incorporate multiple production related factors during cell formation, they have been found to be computationally intractable for realistically sized problems. 
Heuristic approaches have been introduced by Chen [12] and Waghodekar and Sahu [13] binary cell formation problem. Gupta and Devika [14] developed a novel approach to machine-part cell formation using Mahalanobis distance measure.

A number of non-traditional optimsation techniques such as meta heuristics have been investigated by researchers for solving the cell formation problem. Boctor [15], Arkat et al. [16], Jabalameli et al.[17], Ying et al.[18] introduced simulated annealing (SA) based approaches for machine -component grouping. Cheng et al. [19], Paydar and Saidi-Mehrabad [20], Arikaran and Jayabalan [21] used genetic algorithm (GA) based approaches to cell formation problem.. Wu et al.[22] developed tabu search (TS) based approach for machine -part grouping. Zolfaghari and Liang [23]carried out a comparative study of simulated annealing, genetic algorithm and tabu search meta heuristics for solving binary and generalized machine grouping problems Kao and Lin [24] introduced a particle swarm optimization (PSO) based procedure to solve the cell formation problem. Prabhaharan et al. [25] developed an ant colony system (ACS) based cell formation approach and found that ACS performs better than GA. Dimopoulos and Mort [26] solved the cell formation problem using genetic programming (GP) approach. Majority of the above mentioned approaches allow the presence of singleton clusters (cells with single machine) in the solution. Presence of singleton clusters turns the machine-component grouping configuration unrealistic. The appraisal of numerous cell forming approaches indicate that the effectiveness of multi stage solution methodologies based on the integration of traditional and non-traditional optimization techniques have not been investigated adequately. Hence, a multistage heuristic based on the integration of cluster analysis (CA) and simulated annealing techniques has been demonstrated in this paper for generating realistic cell formation solution. The proposed heuristic is designed to offer solutions for both binary and generalized cell formation problems considering processing and production data of components. Component production data considered in the present work are machine requirement, production volume and processing time.

\section{NOTATIONS}

$\begin{array}{ll}a_{i p} & \begin{array}{l}\text { Binary matrix element corresponding to } \\ \text { machine } i \text { and part } p\end{array} \\ a_{j p} & \quad \begin{array}{l}\text { Binary matrix element corresponding to } \\ \text { machine } j \text { and part } p\end{array} \\ c & \text { Index of cells, } c=1,2, \ldots, C \\ d_{i j} & \text { Distance between machines } i \text { and } j \\ e & \text { Number of operations (non-zero entries) within } \\ & \text { the matrix } \\ e_{d} & \text { Number of in-cell operations } \\ e_{o} & \text { Number of out-of-cell operations } \\ e_{v} & \text { Number of voids (0's) within the diagonal } \\ & \text { blocks } \\ d_{i_{c} j_{c}} & \text { Distance between cells } i_{c} \text { and } j_{c},\end{array}$

\begin{tabular}{|c|c|}
\hline$m_{i_{c}}$ & Number of machines in the cell $i_{c}$, \\
\hline$m_{j_{c}}$ & Number of machines in the cell $j_{c}$, \\
\hline$m_{i}$ & Machine index in the cell $i_{c}, m_{i}=1,2,3 \ldots m_{i_{c}}$, \\
\hline$m_{j}$ & Machine index in the cell $j_{c}, m_{j}=1,2,3 \ldots m_{j_{c}}$, \\
\hline$\Omega_{i_{c}}$ & Set of machines in the cell $i_{c}$, \\
\hline$\Omega_{j_{c}}$ & Set of machines in the cell $j_{c}$, \\
\hline$p$ & Part index, $p=1,2, \ldots, N$ \\
\hline$t_{d}$ & Total in-cell processing time \\
\hline$t_{p}^{\max }$ & Maximum processing time of part type $p$ \\
\hline$t_{o}$ & Total out-of-cell processing time \\
\hline$C$ & Total number of cells \\
\hline$L$ & Number of moves at a given temperature $T$ \\
\hline$M$ & Total number of machines in the data set \\
\hline$M_{c}$ & Number of machines in cell $c$ \\
\hline$N$ & Total number of components in the data set \\
\hline$S_{0}$ & Current solution \\
\hline$S_{1}$ & Neighbouring solution \\
\hline$T$ & Temperature coefficient \\
\hline$T_{0}$ & Initial temperature \\
\hline$T_{f}$ & Final temperature or termination criterion \\
\hline$V_{p}$ & Volume of part type $p$ \\
\hline$\alpha$ & Cooling rate \\
\hline$\Gamma$ & Grouping efficacy \\
\hline$\Gamma_{g}$ & Generalised grouping efficacy \\
\hline$\xi\left(S_{0}\right)$ & $\begin{array}{l}\text { Objective function value of the current solution } \\
S_{0}\end{array}$ \\
\hline$\xi\left(S_{1}\right)$ & $\begin{array}{l}\text { Objective function value of the neighbouring } \\
\text { solution } S_{l}\end{array}$ \\
\hline$\Omega_{\mathrm{c}}$ & Set of parts assigned to cell $c$ \\
\hline$\Delta S$ & $\begin{array}{l}\text { Difference between the objective function } \\
\text { values }\end{array}$ \\
\hline
\end{tabular}

\section{DEVELOPMENT OF TWO STAGE CLUSTER ANALYSIS BASED HEURISTIC}

Cluster analysis is a traditional optimization technique which classifies similar objects into relatively homogeneous groups or clusters. In the present work, cluster analysis has been implemented in two stages to obtain a solution which is further improved by simulated annealing phase. The cluster analysis cased heuristic includes the following stages: problem formulation, development of first stage clustering procedure (CA1) and development of second stage clustering procedure (CA2). Initial machinecomponent groups were obtained in the first stage and efficient machine-component groupings were obtained in the second stage of the $\mathrm{CA}$ based heuristic. Grouping efficacy [27] and Generalised grouping efficacy [23] measures are used to measure the goodness of the block diagonalised form of the initial binary and non-binary machine-component incidence matrices corresponding to binary and generalized cell formation problems. A blockdiagonalised matrix contains non-zero entries set in blocks along the diagonal of the matrix indicating the machine groups and part families formed. Non-zero entries outside the diagonal blocks are called as exceptional elements which 
represent the intercellular traffic by components. Higher the exceptional elements higher is the material handling cost. Values for abovementioned goodness measures range from 0 to 1 . Higher the value means more efficient is the machine-component grouping solution formed with lesser intercellular moves by components.

\subsection{Problem Formulation}

Given the binary MCIM, $A_{M \times N}=\left\{a_{x y}\right\}$, for $M$ machines and $N$ components, with regard to binary cell formation problem, obtain the machine-component grouping solution having maximum value for the grouping efficacy measure. Given the non-binary MCIM, $G_{M \times N}=\left\{g_{x y}\right\}$ for $M$ machines and $N$ component with regard the comprehensive cell formation problem with each non-zero matrix entry representing the processing time of a component on a particular machine, and given the volume of each component, obtain the machine-component grouping solution having maximum value for the generalized grouping efficacy measure.

Grouping efficacy $(\Gamma)$ is expressed as:

$$
\Gamma=\frac{1-\psi}{1+\phi}
$$

where

$$
\psi=\frac{e_{0}}{e} \text { and } \phi=\frac{e_{v}}{e}
$$

Generalized grouping efficacy $\left(\Gamma_{g}\right)$ is expressed as:

$$
\Gamma_{g}=\frac{t_{d}}{t_{0}+\sum_{c=1}^{C} M_{c} \sum_{\substack{p=1 \\ p \in \Omega_{c}}}^{N} V_{p} t_{p}^{\max }}
$$

This measure is based on the processing requirements of components, production quantity, and processing or operation times of components on machines.

\subsection{Development of First stage clustering procedure (CA1)}

CA1 intended to obtain initial machine groups and part families in the first stage of the heuristic. CA1 utilises a machine clustering procedure, $\mathrm{MCP}$ to group machines using the first distance measure and a part family formation procedure, PFP for assignment of parts to cells.

\subsubsection{Distance measure for machine grouping}

A distance measure is a measure for understanding the proximity between objects in a dataset. In the present work ,a distance measure shown in equation 3 , which is a special case of Minkowski metric [19] is used as the first distance measure to compute the distance between any two machines $i$ and $j$ for machine grouping:

$$
d_{i j}=\left(\sum_{p=1}^{N}\left|a_{i p}-a_{j p}\right|\right)
$$

Smaller value for $d_{i j}$ indicates machines $i$ and $j$ are similar in processing a set of components and have to be grouped to form a cell to minimize intercellular moves by components. For a non-binary data set with processing times, CP utilizes an equivalent binary matrix formed by replacing non-binary values with 1's (retaining 0's of the matrix in their original locations) to group machines. For the machine cells formed by $\mathrm{CP}$, the corresponding part families are determined by PFP. As per PFP, a part is assigned to a cell such that most of its operations are performed in that cell and improved within-cell machine utilization is achieved.

\subsubsection{Development of Machine clustering procedure (MCP)}

MCP has been developed to group the given set of machines into cells utilizing the information present in the binary. For a non-binary dataset (MCIM) containing processing times, clustering has been carried out utilizing an equivalent binary matrix obtained by replacing the non-binary values in the matrix with 1's and retaining 0's in their original locations. The steps involved in MCP are as follows:

Step 1. Form machine-machine distance square matrix by computing distance values between machine pairs in the binary MCIM using equation 3. Use one half of the matrix as it is symmetrical about the diagonal.

Step 2. Select the least distance value in the matrix. Form the first natural cell (machine group) with associated machine pair $(i j)$.

Step 3. Select the next least value in the distance matrix. Any of the following states may exist with regard to machine pair $(i j)$.

$a$. One of the machines $i$ and $j$ has been already assigned to a cell. In this case, assign the other machine to buffer (a temporary machine list) for its assignment to a specific cell at a later stage.

b. Neither machine $i$ nor machine $j$ has been assigned to an already formed cell and neither of the machines is assigned to the buffer or any one of the machines in the pair is associated with the buffer. In this case, treat the machine pair as a new natural cell.

c. Neither machine $i$ nor machine $j$ is assigned to a previously formed cell and both machines are present in the buffer. In this case, do the following:

$i$. If the number of cells already formed is equal to one, then treat the machine pair as next natural cell.

ii. If the number of cells already formed is greater than one, then 
assign machine $i$ and $j$ to respective cells previously formed where these machines have proximity with one of the machines in those cells with regard to their distance values.

d. Machines $i$ and $j$ have been already assigned to the same cell or two different cells or any one of them to an already formed cell and the other to the buffer. In this case, ignore the distance value.

Repeat step 3 with regard to the remaining distance values in the distance matrix. If the number of machines assigned to cells equals the total number of machines in the dataset, terminate the clustering procedure. Otherwise go to step 4.

Step 4. Assign the remaining unassigned machines in the buffer to those cells where they have close association with one of the machines in those cells with regard to their distance values.

Step 3 a prevents the inclusion of all machines into a single cell. Since machine clusters have been formed around natural machine pairs, singleton cluster formation is avoided.

\subsubsection{Development Part family formation procedure (PFP)}

PFP has been developed to assign part families to machine cells formed by MCP. Steps of PFP are as follows:

Step 1. For each part in the dataset, compute the total number of operations (' 1 'entries in the binary MCIM) performed on machines of each cell.

Step 2. Assign a part to a cell as per the following rules:

$i$. If a part has maximum number of its operations performed in a cell, then assign the part to that cell.

ii. In case of a tie between cells due to equal maximum number of operations, assign that part to a cell with least number of machines to enhance the clustering efficiency through reduction of voids in the block- diagonalised MCIM.

iii. In case of a second tie between cells due to equal least number of machines, follow the following random part assignment scheme to break the tie between cells.

\section{Random part assignment scheme :}

a. Identify cells where tie occurs due to the equal least number of machines. b. Assign the part to the first cell (machine group) with least number of machines.

\subsubsection{Steps for CA1}

The steps involved in CA1 are as follows:

Step 1. Obtain the initial machine groups by implementing the clustering procedure MCP.

Step 2. Assign the part family to each cell as per PFP.

Step 3. Compute the desired cluster goodness measure value of $\left(\Gamma\right.$ or $\left.\Gamma_{g}\right)$.

\subsection{Development Second stage clustering procedure (CA2)}

The Second stage clustering procedure, CA2 of the heuristic has been developed to obtain improved solution in the second stage with superior value for the chosen cluster goodness measure. The procedure for CA2 uses the second distance measure to compute the distance between two cells and PFP.

\subsubsection{Distance measure cell grouping}

The second distance measure used in this work is shown in equation 4 . This measure has been formulated to compute the distance between any two cells $i_{c}$ and $j_{c}$ which have been formed by CA1.

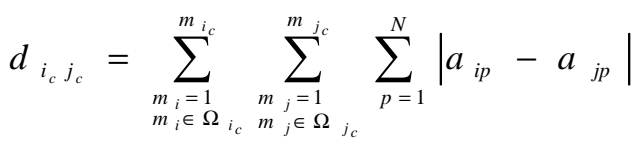

A cell-cell distance square matrix containing the distance values between cell pairs computed using equation 4 has been utilized to obtain the final machine- component configuration. Two cells with smaller $d_{i_{c} j_{c}}$ value indicates the machines within them are similar in processing a group of parts and they can be merged to form a single machine group or cell. In this work, if the total number of cells formed by CA1 in first stage of the heuristic is two, then no regrouping of cells is done. If it is more than two, then the following steps for CA2 have been adopted to regroup the cells. However, the minimum number of cells in such case has been limited to three to prevent the formation of very large cells.

\subsubsection{Steps for CA2}

Steps involved in CA2 are as follows:

Step 1. Form cell-cell distance square matrix by computing the distance between cell pairs based on the binary MCIM using the equation 4. Use one half of the matrix as it is symmetrical about the diagonal.

Step 2. Select the least distance value from the matrix. Represent the associated cell pair $\left(i_{c} j_{c}\right)$ as a cell group and form the single machine group (cell) by combining the machines of cells in the cell pair. 
Step 3. Assign the part families for the revised machine grouping solution as per PFP.

Step 4. Compute the cluster goodness measure value. If it is superior to the value obtained from CA1, then treat the corresponding machine-component groupings as the best solution. Otherwise retain the previous best solution.

Step 5. Select the next least value of the distance between cells from the matrix. Any of the following states may exist with regard to the cell pair $\left(i_{c} j_{c}\right)$ :

a. One of the two cells $i_{c}$ or $j_{c}$ of the associated cell pair $\left(i_{c} j_{c}\right)$ is already included in a cell group. In this case, assign the other cell to this cell group and form a single machine group (cell) by combining the machines of the cells in the cell group.

$b$. None of the cells in the associated cell pair $\left(i_{c} j_{c}\right)$ is found in the already formed cell groups. In this case, consider the cell pair as a new cell group and form a single machine group (cell) by combining the machines of the cells in the cell pair.

$c$. Both cells in the cell pair $\left(i_{c} j_{c}\right)$ have been already assigned to previously formed cell groups. In this case, ignore the distance value.

Step 6. Assign part families to the newly formed machine groupings (cells) using PFP.

Step 7. Compute cluster goodness measure value. If it is better than the earlier value, treat the new machine-component groupings as the best solution. If not, retain the previous best solution.

Step 8. Repeat the steps 5 to 7. If at any instance, the total number of cells assigned to cell groups becomes equal to the total number of cells formed at the end of CA1, terminate the procedure.

\section{DEVELOPMENT OF SIMULATED ANNEALING BASED HEURISTIC}

Simulated annealing [28] is regarded as a popular randomized iterative improvement method for solving combinatorial optimization problems. SA has simple procedure and parameter settings compared to other random search methods. The parameters of annealing schedule of SA include an initial temperature $\left(T_{0}\right)$, final temperature or stopping criterion $\left(T_{f}\right)$, the number of moves or solutions $L$ attempted at each temperature and the rate of cooling $\alpha$ by which temperature is reduced in each iteration of the procedure. In the present work, instead of random initialization, SA is initialized systematically with the best machine grouping solution generated in the first stage by cluster analysis. This solution has been treated as the current solution. The number of cells considered in SA phase is as suggested by the cluster analysis phase. $\Gamma$ and $\Gamma_{g}$ have been treated as objective functions for binary and generalized cell formation problems respectively. To generate a neighbourhood solution from the current solution, a perturbation scheme involving pair wise swapping of machines between cells has been adopted in the present work.

\subsection{Selection of annealing schedule parameter values}

The values for SA parameters $T_{0}, T_{f}, \alpha$, and $L$ may be determined by trial and error proce iven problem. In the present work, these values have been set based on the experimentation using a randomly generated representative data set of 16 machines and 30 components (size $16 \times 30$ ). The performance of the proposed heuristic with regard to $\Gamma_{g}$ has been assessed for 22 random settings of $T_{0}, T_{f}$, and $\alpha$. The value of $L$ has been maintained constant during the experimentation. Based on the results obtained, the values for SA parameters have been finalized as $\quad T_{0}=50.00$, $T_{f}=0.01, \alpha=0.8$, and $L=10$.

\section{PROCEDURE FOR THE PROPOSED MULTI STAGE HEURISTIC}

The detailed procedure for cluster analysis and SA based heuristic is as follows:

Cluster Analysis phase:

First stage:

Step 1. For binary cell formation, input the binary MCIM. For generalized cell formation, input the following:

(a) Non-binary MCIM with processing times as matrix entries.

(b) Equivalent binary MCIM.

(c) Volume of each component.

Step 2. Obtain initial machine-component groups and the chosen cluster goodness measure value by implementing CA1.

Second stage:

Step 3. Obtain the final machine-component groups and the improved value of the chosen cluster goodness measure value by implementing CA2.

Step 4. Return the best value of the chosen cluster goodness measure and the corresponding machine-component groups.

Simulated Annealing phase:

Initialisation: Input the machine groups obtained from the CA phase as $S_{0}$ and represent its cluster goodness measure value as $\xi\left(S_{0}\right)$.

Step 5. Set values for $T_{0}, T_{f}, \alpha$, and $L$.

Step 6. Neighbourhood generation: Generate a neighbouring solution $S_{l}$ by perturbing $S_{0}$. Assign part families and compute $\xi\left(S_{1}\right)$.

Step 7. Neighbourhood search: Compute $\Delta S$ as:

$$
\Delta S=\xi\left(S_{1}\right)-\xi\left(S_{0}\right)
$$


(a) If $\Delta S \geq 0$, accept the candidate solution. Set $S_{0}=S_{l}$.

(b) If $\Delta S<0$, accept the candidate solution if the probability $\exp (\Delta S / T)$ is greater than the random number generated in the range 0 and 1 . Set $S_{0}=S_{1}$. Otherwise reject the candidate solution. Retain $S_{0}$.

Step 8. Repeat steps 5 and 6 for $L$ number of times.

Step 9. Temperature reduction: Update (reduce) the temperature as: $T=T \times \alpha$.

Step 10. Termination: If $T>T_{f}$, go to step 5. Otherwise terminate the search.

Step 11. Return the best objective function value, the corresponding machine-component groupings and the block-diagonalised MCIM.

\section{COMPUTATIONAL EXPERIENCE}

The proposed multistage heuristic has been coded in $\mathrm{C}$ programming language and implemented on a Pentium PC. The performance of the proposed heuristic has been compared with an existing binary cell forming approach known as ZODIAC [7 ] for some binary data sets taken from the literature. $\Gamma$ has been chosen as the performance measure for the comparison. Table 1 shows the results of comparative analysis which proves the superiority of proposed heuristic. The performance of the proposed heuristic has been evaluated for a non-binary data set containing 7 machines and 8 components with components' processing times and volumes as provided by [23]. The solution matrix with three cells obtained by the proposed heuristic is shown in Figure 1. The corresponding value of $\Gamma_{g}$ and that reported by [23] have been found to be $63.93 \%$ and $62.2 \%$ respectively. This proves the ability of the proposed heuristic to capture good quality solution.

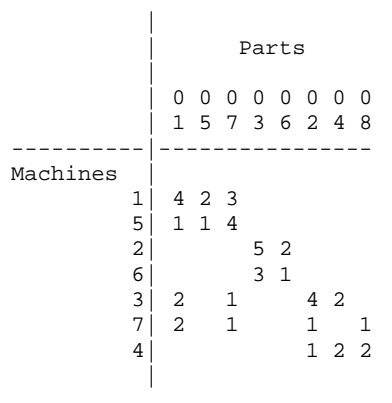

Fig-1: Solution matrix for data set of size $7 \times 8$.
Table-1: Results of the comparative analysis

\begin{tabular}{|c|c|c|c|c|c|}
\hline \multirow{2}{*}{$\begin{array}{c}\text { Data } \\
\text { set } \\
\text { no. }\end{array}$} & \multirow{2}{*}{ Source } & \multirow{2}{*}{$\begin{array}{l}\text { Size } \\
M \times N\end{array}$} & \multirow{2}{*}{$e$} & \multicolumn{2}{|c|}{$\begin{array}{c}\text { Grouping efficacy, } \\
\Gamma(\%) \\
\end{array}$} \\
\hline & & & & ZODIAC & $\begin{array}{l}\text { Proposed } \\
\text { heuristic }\end{array}$ \\
\hline 1 & [29] & $5 \times 7$ & 16 & 56.52 & 62.50 \\
\hline 2 & [4] & $8 \times 20$ & 91 & 58.33 & 58.72 \\
\hline 3 & [9] & $10 \times 20$ & 49 & 100.00 & 100.00 \\
\hline 4 & [15] & $16 \times 30$ & 121 & 34.90 & 48.52 \\
\hline 5 & [15] & $16 \times 30$ & 104 & 58.60 & 59.21 \\
\hline 6 & [15] & $16 \times 30$ & 92 & 68.60 & 70.00 \\
\hline 7 & [15] & $16 \times 30$ & 111 & 26.70 & 45.06 \\
\hline 8 & [15] & $16 \times 30$ & 107 & 72.70 & 72.73 \\
\hline 9 & [15] & $16 \times 30$ & 101 & 76.40 & 76.61 \\
\hline 10 & [15] & $16 \times 30$ & 114 & 32.00 & 56.82 \\
\hline 11 & [30] & $18 \times 24$ & 88 & 41.84 & 47.06 \\
\hline 12 & [30] & $20 \times 35$ & 135 & 75.14 & 75.14 \\
\hline
\end{tabular}

Table-2: Results from CA1, CA2 and SA phases for non-binary data sets

\begin{tabular}{|c|c|c|c|c|c|}
\hline \multirow{3}{*}{$\begin{array}{c}\text { Data } \\
\text { set } \\
\text { no. }\end{array}$} & \multirow{3}{*}{$\begin{array}{l}\text { Size } \\
M \times N\end{array}$} & \multirow{3}{*}{$e$} & \multicolumn{3}{|c|}{$\begin{array}{c}\text { Generalised } \\
\text { grouping efficacy, } \Gamma_{g}(\%) \\
\end{array}$} \\
\hline & & & \multicolumn{2}{|c|}{ CA phase } & \multirow[t]{2}{*}{ SA phase } \\
\hline & & & CA1 & CA2 & \\
\hline 1 & $5 \times 7$ & 14 & 41.44 & 41.44 & 48.10 \\
\hline 2 & $7 \times 11$ & 35 & 45.51 & 45.51 & 49.24 \\
\hline 3 & $8 \times 20$ & 61 & 37.32 & 37.32 & 47.20 \\
\hline 4 & $8 \times 20$ & 64 & 43.94 & 43.94 & 48.23 \\
\hline 5 & $10 \times 20$ & 85 & 34.23 & 35.73 & 42.81 \\
\hline 6 & $10 \times 20$ & 67 & 35.22 & 38.25 & 41.11 \\
\hline 7 & $12 \times 24$ & 96 & 36.55 & 36.55 & 38.12 \\
\hline 8 & $12 \times 24$ & 113 & 34.17 & 34.17 & 38.55 \\
\hline 9 & $16 \times 30$ & 187 & 29.68 & 29.68 & 34.66 \\
\hline 10 & $20 \times 35$ & 174 & 30.76 & 31.55 & 34.08 \\
\hline
\end{tabular}




\section{CONCLSIONS}

The development of a multi stage cell formation heuristic based on the integration of cluster analysis and simulated annealing techniques has been described in this paper. The proposed heuristic is capable of offering realistic solutions free of singleton clusters for binary as well as generalised cell formation problems. The number of cells to be formed is suggested by the heuristic and it need not be specified in advance by the practitioner arbitrarily. Computational results for moderately sized data sets indicate that the proposed methodology based on the integration of traditional and non-traditional optimization techniques provides promising quality solutions for binary and nonbinary matrices. This framework can be further extended to incorporate performance measures which include production parameters other than those considered in this work.

\section{REFERENCES}

[1] Grammatoula Papaioannou and John M. Wilson, "The evolution of cell formation problem methodologies based on recent studies (1997-2008): Review and directions for future research", European Journal of Operational Research, Vol. 206, pp. 509-521, 2010.

[2] P. K Arora, A. Haleem and M. K. Singh, "Recent development of cellular manufacturing systems", $\mathrm{Sa}^{-}$dhana ${ }^{-}$, Vol.38, Part 3, pp. 421-428, June 2013.

[3] Burbidge, J.L., "Production Flow Analysis", Production Engineer, Vol. 42, pp. 742-752, 1963.

[4] Chandrasekharan, M.P. and Rajagopalan R., "MODROC: An extension of rank order clustering for group technology", International Journal of Production Research, Vol. 24, No.5, pp. 1221-1233, 1986.

[5] McAuley, J., "Machine grouping for efficient production”, Production Engineer, Vol. 51, pp. 53-57, 1972.

[6] Gupta, T. and Seifoddini, H., "Production data based similarity coefficient for machine-component grouping decisions in design of a cellular manufacturing system", International Journal of Production Research, Vol. 28, No.7, pp. 1247-1269, 1990.

[7] Chandrasekharan, M.P. and Rajagopalan R., "ZODIAC - an algorithm for concurrent formation of part families and machine cells", International Journal of Production Research, Vol. 25, No. 6, pp. 835-850, 1987.

[8] Srinivasan, G. and Narendran, T.T., "GRAFICS - a non-hierarchical clustering algorithm for group technology", International Journal of Production Research, Vo. 29, No.3, pp. 463-478, 1991.

[9] Srinivasan, G., Narendran, T.T. and Mahadevan, B., "An assignment model for the part-families problem in group technology", International Journal of Production Research, Vol. 28, No. 1, pp. 145-152, 1990.

[10] Mohammad Mahdi Paydar and Navid Sahebjamnia, "Designing a mathematical model for cell formation problem using operation sequence", Journal of
Applied Operational Research,

Vol.1, No.1, pp. 30-38, 2009.

[11] I. Mahdavi, M. M. Paydar, M. Solimanpur and M. Saidi-Mehrabad,"A Mathematical Model for Integrating Cell Formation Problem with Machine Layout", International Journal of Industrial Engineering \& Production Research, Vol.21, No.2, pp 61-70, September 2010.

[12] Chen, M.C., "Configuration of cellular manufacturing systems using association rule induction", International Journal of Production Research, Vol.41, No.2, pp. 381-395, 2003.

[13] Srinivasan,G., Narendran, T.T., \& Mahadevan, B., "An assignment model for the part-families problem in group technology", International Journal of Production Research, Vol. 28, No.1, 145-152, 1990.

[14] N. Srinivasa Gupta and D. Devika, "A Novel Approach to Machine-Part Cell Formation Using Mahalanobis Distance", European Journal of Scientific Research, Vol.62, No.1, pp. 43-47, 2011.

[15] Boctor, F.F., "A linear formulation of the machinepart cell formation problem", International Journal of Production Research, Vol. 29, No.2, pp. 343-356,1991.

[16] Arkat, J., Saidi, M. and Abbasi, B., “Applying simulated annealing to cellular manufacturing system design", International Journal of Advanced Manufacturing Technology, Vo.32, pp.531-536, 2007.

[17] Jabalameli, M.S., Arkat, J. and Sakri, M.S., "Applying meta heuristics in the generalized cell formation problem considering machine reliability", Journal of the Chinese Institute of Industrial Engineers, Vo. 25, pp. 261-274, 2008.

[18] Kuo-Ching Ying, Shih-Wei Lin and Chung-Cheng $\mathrm{Lu}$, "Cell formation using a simulated annealing algorithm with variable neighbourhood", European Journal of Industrial Engineering, Vol.5, No.1, pp. 22-42, 2011.

[19] Cheng, C.H., Gupta, Y. P., Lee, W.H. and Wong, K.F., "A TSP-based heuristic for forming machine groups and part families", International Journal of Production Research, Vo. 36, No. 5, pp.1325$1337,1998$.

[20] Mohammad Mahdi Paydar and MohammadSaidiMehrabad, "A hybrid genetic-variable neighborhood search algorithm for the cell formation problem based on grouping efficacy", Computers \& Operations Research,Vol.40 pp. 980990,2013.

[21] P. Arikaran ,V. Jayabalan, "A Grouping Genetic Algorithm for Solving the Machine Component Grouping Problem", European Journal of Scientific Research, Vol.63, No.3, pp. 347-357, 2011.

[22] Tai-Hsi Wu, Jinn-Yi Yeh, Chin-Chih Chang, “A hybrid Tabu Search Algorithm to Cell Formation Problem and its Variants", World Academy of 
Science, Engineering and Technology, 53, pp.10901094, 2009.

[23] Zolfaghari, S. and Liang, M., "Comparative study of simulated annealing, genetic algorithm and tabu search for solving binary and comprehensive machine-grouping problems", International Journal of Production Research, Vol.40, No.9, pp. 2141-2158, 2002.

[24] Yucheng Kao and Chia-Hsien Lin, "A PSO-based approach to cell formation problems with alternative process routings", International Journal of Production Research, Vol. 50, No. 15, pp. 4075-4089, August 2012.

[25] Prabhaharan, G., Muruganandam, A, Asokan, P., Girish, B.S.,"Machine cell formation for cellular manufacturing systems using an ant colony system approach", International Journal of Advanced Manufacturing Technology, Vol. 25, pp. 1013-1019, 2005.

[26] Dimopoulos, C. and Mort, N., "A hierarchical clustering methodology based on genetic programming for the solution of simple cellformation problems", International Journal of Production Research, Vol. 39, No.1, pp. 1-19, 2001.

[27] Kumar, C.S. and Chandrasekharan, M.P., "Grouping Efficacy: a quantitative criterion for goodness of block diagonal forms of binary matrices in group technology", International Journal of Production Research, Vol.28, No.2, pp. 233-243, 1990.

[28] Kirkpatrick, S., Gelati, Jr, C.D. and Vecchi, M.P., "Optimization by simulated annealing ", Science, Vol. 220, pp. 671-680, 1983.

[29] King, J.R. and Nakornchai, V., "Machine component group formation in group technology: review and extension", International Journal of Production Research, Vol. 20, pp. 117-133, 1982.

[30] Carrie, A.S., "Numerical taxonomy applied to group technology and plant layout", International Journal of Production Research, Vol. 11, pp. 399-416, 1973. 